\title{
Fitness is Recovered with the Decline of Dimethachlon Resistance in Laboratory- induced Mutants of Sclerotinia sclerotiorum after Long-term Cold Storage
}

\author{
Jin-Li Li ${ }^{1}$, Feng-Ci $\mathrm{Wu}^{2}$ and Fu-Xing $\mathrm{Zhu}^{1 *}$ \\ ${ }^{1}$ College of Plant Science and Technology, Huazhong Agricultural University, Wuhan 430070, China \\ ${ }^{2}$ Agro-Biotechnology Institute of Jilin Academy of Agricultural Sciences, Changchun 130033, China
}

(Received on April 27, 2015; Revised on June 18, 2015; Accepted on July 5, 2015)

\begin{abstract}
After four years of cold storage, dimethachlon resistance of two laboratory-induced resistant Sclerotinia sclerotiorum isolates SCG7 and LA50 declined by 99.5\% and $98.9 \%$, respectively, and cross resistance to iprodione and procymidone also declined dramatically. Along with the decline of fungicide resistance, osmotic sensitivity to sodium chloride and glucose decreased tremendously; mycelial growth rate, sclerotia number and weight per potato dextrose agar (PDA) plate increased on average by $118.6 \%, 85.5 \%$ and $64.5 \%$, respectively; and virulence to detached leaves of oilseed rape increased by $72.7 \%$ on average. Significant negative correlations were detected between dimethachlon resistance levels and mycelial growth rate on PDA (r = $-0.980, P=0.021$ ), and between resistance levels and lesion diameters on detached leaves of oilseed rape plants $(\mathrm{r}=-\mathbf{0 . 9 9 7}, \boldsymbol{P}=\mathbf{0 . 0 0 2})$. These results have profound implications for assessing the potential risk for resistance development to dicarboximide fungicides in $S$. sclerotiorum.
\end{abstract}

Keywords : cold storage, dimethachlon resistance, fitness penalty, Sclerotinia sclerotiorum

Sclerotinia sclerotiorum (Lib.) de Bary is a necrotrophic plant pathogen capable of infecting more than 400 species of plants worldwide including many economically important oil crops and vegetables, such as oilseed rape, soybean, peanut, sunflower, and lettuce (Boland and Hall, 1994; Bolton et al., 2006). Sclerotinia stem rot (SSR) on oilseed rape caused by this pathogen has traditionally been difficult to control because disease-resistant cultivars have not yet been sufficiently developed (Lu, 2003). Applica-

*Corresponding author.

Phone) +86-27-87286991, FAX) +86-27-87384670

E-mail) zhufuxing@mail.hzau.edu.cn tion of fungicide is still the principal method for control of SSR in China (Wang et al., 2014). More than a decade of applications of the dicarboximide fungicide dimethachlon have resulted in low levels of resistance to dimethachlon in Jiangsu Province of eastern China (Ma et al., 2009). More recently, medium to high levels of dimethachlon resistance have been reported in Shaanxi Province of northwestern China, Heilongjiang Province of northeastern China and Hunan Province of central China (Zhou et al., 2014a, b).

The evolution of fungicide resistance will be slower if resistant isolates have lower saprophytic and pathogenic fitness, and/or higher phenotypic instability (Bardas et al., 2008). Fitness parameters associated with fungicide resistance have been extensively studied in S. sclerotiorum and other ascomycete plant pathogens. Previous studies demonstrated that for dimethachlon-resistant field isolates of $S$. sclerotiorum, mycelial growth on PDA media and virulence to oilseed rape plants decreased significantly, and osmotic sensitivity to glucose and sodium chloride $(\mathrm{NaCl})$ increased compared to dimethachlon-sensitive isolates (Zhou et al., 2014a). Laboratory-induced dimethachlonresistant isolates of $S$. sclerotiorum also exhibited reduced mycelial growth on PDA media and increased osmotic sensitivity to glucose and $\mathrm{NaCl}$, but no significant difference in virulence was found compared to field sensitive isolates (Ma et al., 2009).

Instability of fungicide resistance after long-term storage has been investigated in a variety of fungicide-phytopathogen combinations. Resistance to the de-methylation inhibitor (DMI) fungicide propiconazole in Monilinia fructicola decreased remarkably after cold storage at $5^{\circ} \mathrm{C}$ for 8 and 34 months (Cox et al., 2007). Different storage methods, whether with mycelia or conidia of $M$. fructicola at different temperatures $\left(4,-20\right.$ and $\left.-80^{\circ} \mathrm{C}\right)$, could not prevent the decline of propiconazole resistance in the resistant isolates (Zhu et al., 2012). Resistance to the DMI fungicide flutriafol in Cercospora beticola became unstable after cold storage at $3^{\circ} \mathrm{C}$ for 6 and 10 months, but did not decline after 10 
successive transfers on artificial culture media (Karaoglanidis and Thanassoulopoulos, 2002).

Phenotypic instability and fitness penalty associated with fungicide resistance have much relevance to the development as well as management of fungicide resistance. Up to present, no report has been published about the effects of long-term cold storage on stability of fungicide resistance in S. sclerotiorum. Therefore, the objectives of this study were to assess effects of long-term cold storage on stability of dimethachlon resistance and fitness such as mycelial growth rate, sclerotia production and osmotic sensitivity in S. sclerotiorum.

Field isolates of $S$. sclerotiorum used in this study were collected from diseased oilseed rape plants in 2009. Dimethachlon-induced resistant isolates were obtained by growing field sensitive isolates successively on dimethachlonamended PDA media. Sclerotia of each isolate produced on PDA plates were air dried and placed in $1.5 \mathrm{ml}$ centrifuge tubes. The tubes were sealed with Parafilm sealing film and stored at $4^{\circ} \mathrm{C}$ in a refrigerator for 4 years. The $50 \%$ effective concentration (EC50) values were determined according to Zhou et al. (2014a) based on inhibitions of mycelial growth on PDA supplemented with fungicide. For determination of mycelial growth, sclerotia number and weight, PDA plates inoculated with mycelial plugs (5 $\mathrm{mm}$ in diameter) were incubated at $25^{\circ} \mathrm{C}$ in the dark in a growth chamber. After $48 \mathrm{~h}$ of incubation, diameter of each colony was measured at right angles. At the end of 21 days of incubation, the number of sclerotia on each PDA plate was recorded. All the sclerotia in each dish were weighed after drying at $50^{\circ} \mathrm{C}$ for two days. For determination of osmotic sensitivity to sodium chloride $(\mathrm{NaCl})$ and glucose, mycelial plugs were transferred onto PDA media amended with $\mathrm{NaCl}$ or glucose at different concentrations. PDA media free of $\mathrm{NaCl}$ and glucose were used as the control. Six plates for each concentration of $\mathrm{NaCl}$ and glucose were incubated at $25^{\circ} \mathrm{C}$ in the dark for $48 \mathrm{~h}$. Diameter of each fungal colony was measured at right angles. Percent inhibitions of mycelial growth relative to the $\mathrm{NaCl}$-glucose-free control were calculated. For determination of virulence to detached leaves of oilseed rape, leaves of similar size (10 to $13 \mathrm{~cm}$ in diameter) were detached from approximately 6-week-old oilseed rape plants, rinsed with sterile water, transferred to $15-\mathrm{cm}$ petri dishes lined with wet filter paper to maintain high humidity and then inoculated on the adaxial surface with mycelial plugs. The inoculated leaves were incubated in a growth chamber $\left(25^{\circ} \mathrm{C}\right.$, photoperiod of $16 \mathrm{~h}$ ). Diameters of disease lesions (necrotic area) were measured at right angles after $48 \mathrm{~h}$ of incubation. There were 6 replicates for each isolate. All experiments were repeated independently once. Student's t-test in SPSS (Statistical Product and Service Solutions, ver. 19.0, SPSS Inc., Chicago, IL) indicated that there were no significant differences $(P>0.05)$ between experiments, hence data from the two repeated experiments were pooled together for further analyses. Two tailed paired Student's t-test in SPSS at $\alpha=0.05$ was employed to test significant differences in resistance levels and biological characteristics such as mycelial growth rate and sclerotia production between before and after long-term cold storage. The EC50 values were logarithm-transformed before further statistical analysis because logarithmic transformation could improve accuracy and reliability of statistical analysis of EC50 values (Liang et al., 2015).

Results showed that compared with the initial EC50 values before cold storage, dimethachlon resistance of the two isolates SCG7 and LA50 after four years of cold storage declined by $99.5 \%$ and $98.9 \%$, respectively (Table 1). EC50 value for isolate SCG7 after storage was only $0.48 \mu \mathrm{g} / \mathrm{ml}$, indicating that resistance to dimethachlon had almost completely disappeared. There was an apparent positive cross resistance between dimethachlon and two

Table 2. Cross-resistance to iprodione and procymidone in dimethachlon-induced resistant isolates of Sclerotinia sclerotiorum before and after four years of cold storage

\begin{tabular}{lcccc}
\hline \multirow{2}{*}{ Isolate } & \multirow{2}{*}{$\begin{array}{c}\text { Before/After } \\
\text { cold storage }\end{array}$} & \multicolumn{3}{c}{ EC50 value $(\mu \mathrm{g} / \mathrm{ml})$} \\
\cline { 3 - 5 } & & Dimethachlon & Iprodione & Procymidone \\
\hline \multirow{2}{*}{ SCG7 } & Before & 97.69 & $>100$ & $>200$ \\
& After & 0.48 & 0.18 & 0.13 \\
\hline \multirow{2}{*}{ LA50 } & Before & 178.49 & $>100$ & $>200$ \\
& After & 1.84 & 0.32 & 0.18 \\
\hline
\end{tabular}

Table 1. Decline of dimethachlon resistance in laboratory-induced isolates of Sclerotinia sclerotiorum after four years of cold storage

\begin{tabular}{ccccc}
\hline Isolate & EC50 before storage $(\mu \mathrm{g} / \mathrm{ml})$ & EC50 after storage $(\mu \mathrm{g} / \mathrm{ml})$ & Decline of resistance $(\%)$ & $P$ value by paired $t$-test $^{\mathrm{a}}$ \\
\hline SCG7 & 97.69 & 0.48 & 99.5 & 0.024 \\
LA50 & 178.49 & 1.84 & 98.9 & 0.9 \\
\hline
\end{tabular}

aPaired t-test for significant difference in dimethachlon resistance between before and after cold storage was conducted after EC50 values were logarithmically transformed. 
Table 3. Mycelial growth and sclerotia production of dimethachlon-induced resistant isolates of Sclerotinia sclerotiorum before and after four years of cold storage

\begin{tabular}{|c|c|c|c|c|c|c|}
\hline Parameter & Isolate & $\begin{array}{l}\text { Before } \\
\text { storage }\end{array}$ & $\begin{array}{c}\text { After } \\
\text { storage }\end{array}$ & $\begin{array}{c}\text { Percent } \\
\text { increase }(\%)\end{array}$ & $\begin{array}{c}\text { Average } \\
\text { increase }(\%)\end{array}$ & $\begin{array}{c}P \text { value by } \\
\text { paired } t \text {-test }\end{array}$ \\
\hline \multirow{2}{*}{$\begin{array}{l}\text { Mycelial growth } \\
(\mathrm{cm})^{\mathrm{a}}\end{array}$} & SCG7 & 4.52 & 8.35 & 84.7 & \multirow{2}{*}{118.6} & \multirow{2}{*}{$<0.001$} \\
\hline & LA50 & 2.80 & 7.07 & 152.5 & & \\
\hline \multirow{2}{*}{ Number of sclerotia ${ }^{b}$} & SCG7 & 10.0 & 26.70 & 167.0 & \multirow{2}{*}{85.5} & \multirow{2}{*}{0.001} \\
\hline & LA50 & 15.40 & 16.00 & 3.9 & & \\
\hline \multirow{2}{*}{$\begin{array}{l}\text { Sclerotia dry weight } \\
(\mathrm{mg})^{\mathrm{c}}\end{array}$} & SCG7 & 47.2 & 110.9 & 134.9 & \multirow{2}{*}{64.5} & \multirow{2}{*}{0.247} \\
\hline & LA50 & 119.3 & 112.1 & -6.0 & & \\
\hline
\end{tabular}

${ }^{\mathrm{a}}$ Mycelial growth was measured after $48 \mathrm{~h}$ of incubation on PDA plates at $25^{\circ} \mathrm{C}$ in the dark.

${ }^{b}$ Number represents the average number of sclerotia per plate calculated from 6 replicates.

'Sclerotia weight represents the average weight of sclerotia per plate.

${ }^{\mathrm{d}}$ Paired t-test was conducted to compare the measured parameters before and after four years of cold storage.

other dicarboximide fungicides iprodione and procymidone (Table 2). After four years of cold storage, along with the drastic decline of resistance to dimethachlon, resistance to iprodione and procymidone also decreased considerably. On average, mycelial growth on PDA media of the two isolates increased by $118.6 \%$, number of sclerotia per plate increased by $85.5 \%$ and sclerotia dry weight per plate increased by $64.5 \%$ (Table 3 ). These results demonstrated that along with the tremendous decline of resistance to dimethachlon after long-term cold storage, vegetative fitness parameters mycelial growth and sclerotia production increased remarkably.

After four years of cold storage, sensitivity of isolates SCG7 and LA50 to $\mathrm{NaCl}$ and glucose decreased substantially (Fig. 1). Percent inhibition of mycelial growth of SCG7 and LA50 on PDA media amended with $\mathrm{NaCl}$ at 20 $\mathrm{g} / \mathrm{L}$ decreased from $100.0 \%$ and $73.1 \%$ before cold storage to $8.5 \%$ and $29.4 \%$ after cold storage, respectively. Com- pared with virulence before cold storage, lesion diameters on detached leaves of oilseed rape for isolates SCG7 and LA50 after cold storage increased by $84.8 \%$ and $61.3 \%$, respectively (Fig. 2A). There was a statistically significant negative correlation $(\mathrm{r}=-0.997, P=0.002)$ between logarithms of EC50 values and lesion diameters on detached leaves of oilseed rape (Fig. 2B).

Stability of fungicide resistance may vary with pathogen species, fungicide or storage method. Botrytis cinerea resistance to pyraclostrobin, boscalid and cyprodinil was reported to be stable after successive transfers on artificial media (Bardas et al., 2008; Kim and Xiao, 2011). Dimethachlon resistance in $S$. sclerotiorum decreased considerably after transfers on PDA for 20 consecutive generations (data not shown). Studies on flutriafol-resistant $C$. beticola isolates demonstrated that after successive transfers, resistance did not change significantly. However, after fungal strains had been exposed to cold conditions for six to ten months,
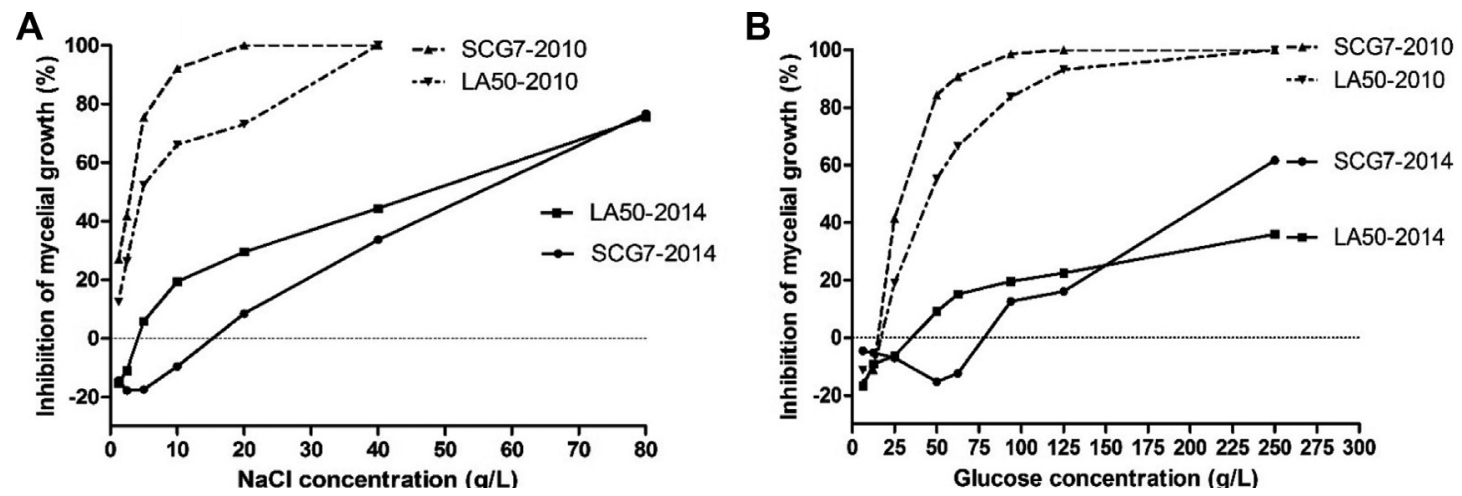

Fig. 1. Osmotic sensitivity of two laboratory-induced resistant isolates LA50 and SCG7 of Sclerotinia sclerotiorum before and after four years of cold storage. (A) sensitivity to $\mathrm{NaCl}$; (B) sensitivity to glucose. In each figure, the two dashed lines (SCG7-2010 and LA502010) represent data before storage and the two solid lines (SCG7-2014 and LA50-2014) represent data after storage. 
A

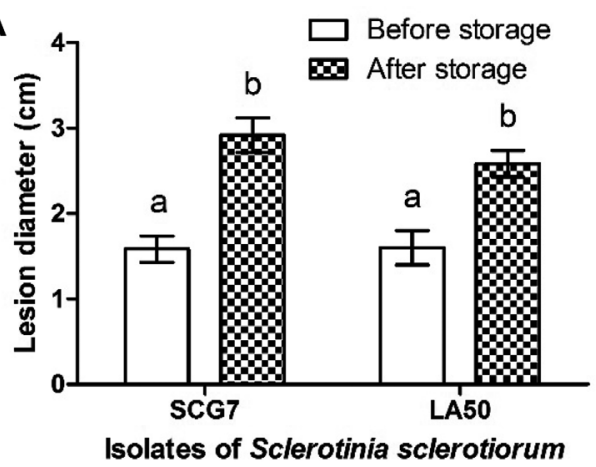

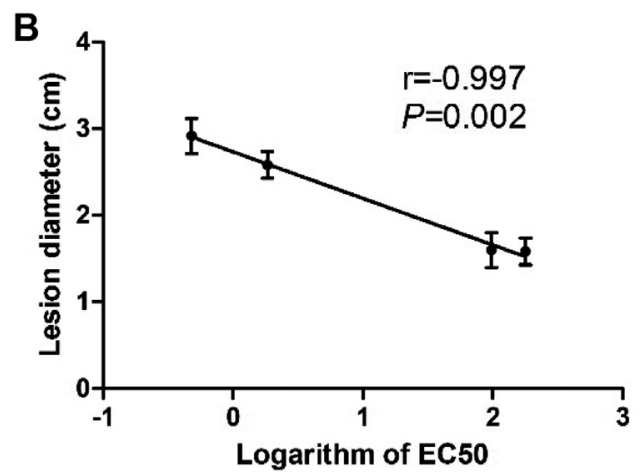

Fig. 2. Virulence to detached leaves of oilseed rape plants and correlation between virulence and resistance to dimethachlon in isolates SCG7 and LA50 of Sclerotinia sclerotiorum. (A) Virulence to oilseed rape plants before and after four years of cold storage. Error bar represents standard errors of 12 replicates. (B) Correlation between virulence and logarithms of EC50 values for dimethachlon.

flutriafol resistance decreased remarkably (Karaoglanidis and Thanassoulopoulos, 2002).

Studies on B. cinerea showed that heterokaryosis might be related to instability of resistance to the dicarboximide fungicide vinclozolin (Yourman et al., 2001). Fungicide-resistant heterokaryons of $B$. cinerea might contain both sensitive and resistant nuclei and the ratio of the two nucleus types might be modified under different conditions (Faretra and Pollastro, 1993). It is well known that S. sclerotiorum is multinucleate (Bolton et al., 2006). Heterokaryosis may in part account for the instability of dimethachlon resistance.

It had been postulated that fitness costs might be associated more frequently with polygene-controlled resistance than with monogenic-controlled resistance (Bardas et al., 2008). Resistance to carbendazim in S. sclerotiorum was caused by point mutations in $\beta$-tubulin gene, i.e. E198A for high-level resistance and F200Y for medium-level resistance (Li et al., 2003; Yin et al., 2010). In contrast, dimethachlon resistance in $S$. sclerotiorum seemed to be gradual rather than discrete, suggesting that dimethachlon resistance might be controlled by more than one gene (Duan et al., 2014; Ma et al., 2009; Zhou et al. 2014a). Thus, fitness penalties associated with dimethachlon resistance may be related to the possible multiple mechanisms and polygene that involved in dicarboximide resistance in S. sclerotiorum.

The present study demonstrated that there were statistically significant negative correlations between dimethachlon resistance levels and mycelial growth, and between resistance and virulence to detached leaves of oilseed rape. The negative correlations between dimethachlon resistance levels and fitness parameters may be attributed to pleiotropy of resistance genes. Studies by Duan et al. (2013) showed that disruption of the class III histidine kinases gene Shkl of S. sclerotiorum resulted in resistance to dimethachlon and fludioxonil, and enhanced sensitivity to osmotic stress and oxidative stress. The Shkl mutant showed a significant reduction in vegetative hyphal growth as well, but no changes in virulence were observed.

Results of the present study are valuable for assessing and understanding the potential risk for dimethachlon resistance development under field conditions. The fitness costs associated with dimethachlon resistance may partially account for the slow development of dimethachlon resistance in the fields given the fact that dimethachlon has been used to control S. sclerotiorum for more than a decade. These results also imply that discontinuing use of dimethachlon may help reduce dimethachlon resistance frequency under field conditions. Further studies are needed to monitor development of dicarboximide fungicide resistance in this devastating plant pathogen.

\section{Acknowledgments}

This study was supported by the Special Fund for Agroscientific Research in the Public Interest (No. 201103016) and National Natural Science Foundation of China (No. 31371964). We would like to thank Prof. Daohong Jiang of Huazhong Agricultural University for technical support.

\section{References}

Bardas, G. A., Myresiotis, C. K. and Karaogllanidis, G. S. 2008. Stability and fitness of anilinopyrimidine-resistant strains of Botrytis cinerea. Phytopathology 98:443-450.

Boland, G. J. and Hall, R. 1994. Index of plant hosts of Sclerotinia sclerotiorum. Can. J. Plant Pathol. 16:93-108.

Bolton, M. D., Thomma, B. P. and Nelson, B. D. 2006. Sclero- 
tinia sclerotiorum (Lib.) de Bary: biology and molecular traits of a cosmopolitan pathogen. Mol. Plant Pathol. 7:1-16.

Cox, K. D., Bryson, P. K. and Schnabel, G. 2007. Instability of propiconazole resistance and fitness in Monilinia fructicola. Phytopathology 97:448-453.

Duan, Y. B., Ge, C. Y., Liu, S. M., Wang, J. X. and Zhou, M. G. 2013. A two-component histidine kinase Shk1 controls stress response, sclerotial formation and fungicide resistance in Sclerotinia sclerotiorum. Mol. Plant Pathol. 14:708-718.

Duan, Y. B., Ge, C. Y. and Zhou, M. G. 2014. Molecular and biochemical characterization of Sclerotinia sclerotiorum laboratory mutants resistant to dicarboximide and phenylpyrrole fungicides. J. Pest Sci. 87:221-230.

Faretra, F. and Pollastro, S. 1993. Genetics of sexual compatibility and resistance to benzimidazole and dicarboximide fungicides in isolates of Botryotinia fuckliana (Botrytis cinerea) from nine countries. Plant Pathol. 42:48-57.

Karaoglanidis, G. S. and Thanassoulopoulos, C. C. 2002. Phenotypic instability of Cercospora beticola sacc. strains expressing resistance to the sterol demethylation-inhibiting (DMI) fungicide flutriafol after cold exposure. J. Phytopathol. 150:692-696.

Kim, Y. K. and Xiao, C. L. 2011. Stability and fitness of pyraclostrobin- and boscalid-resistant phenotypes in field isolates of Botrytis cinerea from apple. Phytopathology 101:1385-1391.

Li, H. X., Lu, Y. J., Zhou, M. G. and Wang, X. F. 2003. Mutation in $\beta$-tubulin of Sclerotinia sclerotiorum conferring resistance to carbendazim in rapeseed field isolates. Chin. J. Oil Crop Sci. 25:56-60.

Liang, H. J., Li, J. L., Di, Y. L., Zhang, A. S. and Zhu, F. X. 2015. Logarithmic transformation is essential for statistical analysis of fungicide EC50 values. J. Phytopathol. 163:456-464.
Lu, G. 2003. Engineering Sclerotinia sclerotiorum resistance in oilseed crops. African J. Biotech. 2:509-516.

Ma, H. X., Feng, X. J., Chen, Y., Chen, C. J. and Zhou, M. G. 2009. Occurrence and characterization of dimethachlon insensitivity in Sclerotinia sclerotiorum in Jiangsu Province of China. Plant Dis. 93:36-42.

Reimann, S. and Deising, H. B. 2005. Inhibition of efflux transporter-mediated fungicide resistance in Pyrenophora tritici-repentis by a derivative of 4'-hydroxyflavone and enhancement of fungicide activity. Appl. Environ. Microbiol. 71:3269-3275.

Wang, Y., Hou, Y. P., Chen, C. J. and Zhou, M. G. 2014. Detection of resistance in Sclerotinia sclerotiorum to carbendazim and dimethachlon in Jiangsu Province of China. Australas. Plant Pathol. 43:307-312.

Yin, Y. N., Liu, X., Shi, Z. Q. and Ma, Z. H. 2010. A multiplex allele-specific PCR method for the detection of carbendazimresistant Sclerotinia sclerotiorum. Pestic. Biochem. Physiol. 97:36-42.

Yourman, L. F., Jeffers, S. N. and Dean, R. A. 2001. Phenotype instability in Botrytis cinerea in the absence of benzimidazole and dicarboximide fungicides. Phytopathology 91:307-315.

Zhou, F., Zhang, X. L., Li, J. L. and Zhu, F. X. 2014a. Dimethachlon resistance in Sclerotinia sclerotiorum in China. Plant Dis. 98:1221-1226.

Zhou, F., Zhu, F. X., Zhang, X. L. and Zhang, A. S. 2014b. First report of dimethachlon resistance in field isolates of Sclerotinia sclerotiorum on oilseed rape in Shaanxi Province of northwestern China. Plant Dis. 98:568.

Zhu, F. X., Bryson, P. K. and Schnabel, G. 2012. Influence of storage approaches on instability of propiconazole resistance in Monilinia fructicola. Pest Manag. Sci. 68:1003-1009. 